\title{
Large $|k|$ behavior of d-bar problems for domains with a smooth boundary
}

\author{
Christian Klein Johannes Sjöstrand Nikola Stoilov
}

To Ari Laptev on the occasion of his 70th birthday

\begin{abstract}
In a previous work on the large $|k|$ behavior of complex geometric optics solutions to a system of d-bar equations, we treated in detail the situation when a certain potential is the characteristic function of a strictly convex set with realanalytic boundary. We here extend the results to the case of sets with smooth boundary, by using almost holomorphic functions.
\end{abstract}

\section{Introduction}

This note is concerned with solutions to the Dirac system

$$
\left\{\begin{array}{l}
\bar{\partial} \phi_{1}=\frac{1}{2} q \mathrm{e}^{\bar{k} \bar{z}-k z} \phi_{2} \\
\partial \phi_{2}=\sigma \frac{1}{2} \bar{q} \mathrm{e}^{k z-\bar{k} \bar{z}} \phi_{1}, \quad \sigma= \pm 1
\end{array}\right.
$$

subject to the asymptotic conditions

$$
\lim _{|z| \rightarrow \infty} \phi_{1}=1, \quad \lim _{|z| \rightarrow \infty} \phi_{2}=0
$$

where $q=q(x, y)$ is a complex-valued field, the spectral parameter $k \in \mathbb{C}$ is independent of $z=x+\mathrm{i} y$, and

$$
\partial:=\frac{1}{2}\left(\frac{\partial}{\partial x}-\mathrm{i} \frac{\partial}{\partial y}\right) \quad \text { and } \quad \bar{\partial}:=\frac{1}{2}\left(\frac{\partial}{\partial x}+\mathrm{i} \frac{\partial}{\partial y}\right) .
$$

C. Klein: Institut de Mathématiques de Bourgogne, UMR 5584

Université de Bourgogne-Franche-Comté, 9 avenue Alain Savary, 21078 Dijon Cedex, France; email: Christian.Klein@u-bourgogne.fr

J. Sjöstrand: Institut de Mathématiques de Bourgogne, UMR 5584

Université de Bourgogne-Franche-Comté, 9 avenue Alain Savary, 21078 Dijon Cedex, France; email: Johannes.Sjostrand@u-bourgogne.fr

N. Stoilov: Institut de Mathématiques de Bourgogne, UMR 5584

Université de Bourgogne-Franche-Comté, 9 avenue Alain Savary, 21078 Dijon Cedex, France; email: Nikola.Stoilov@u-bourgogne.fr 
The functions $\phi_{i}(z ; k), i=1,2$ depend on $z$ and $k$ where it is understood that they need not be holomorphic in either variable. This system appears in the scattering theory of the integrable Davey-Stewartson II equation [2] and in the context of Calderón's problem [1] (for more applications and references, see [4]).

In [4], we have addressed the large $|k|$ behavior of the solutions to (1.1) and (1.2). It was shown that the $k$ dependence for large $|k|$ strongly depends on the regularity of the potential $q$ in (1.1). Of particular interest is the case of potentials being the characteristic function of a compact domain with strictly convex boundary. It was shown in [4] that in this case $\phi_{2}=\mathscr{O}\left(|k|^{-1 / 2}\right)$ in polynomially weighted $L^{2}$-spaces. The leading order contribution to $\phi_{2}$ is equal to $\overline{f(z, k)} /(2 \pi)$, where $f$ is given by the integral

$$
f(z, k)=\int_{\Omega} \frac{1}{z-w} e^{\overline{k w}-k w} L(\mathrm{~d} w)=\iint_{\Omega} \frac{e^{\overline{k w}-k w}}{z-w} \frac{\mathrm{d} \bar{w} \wedge \mathrm{d} w}{2 i},
$$

for $z, k \in \mathbb{C},|k| \gg 1$. Here

$$
L(\mathrm{~d} w)=\mathrm{d} \operatorname{Re}(w) \wedge \mathrm{d} \operatorname{Im}(w)=\frac{\mathrm{d} \bar{w} \wedge \mathrm{d} w}{2 i}
$$

is the Lebesgue measure. The large $|k|$ asymptotics of $f$ was computed in [4] when $\partial \Omega$ is analytic.

In this note we extend the results of Section 5 in [4] to the case of strictly convex domains $\Omega \Subset \mathbb{C}$ with a boundary that is smooth but not necessary analytic. In doing so, we follow the complex analysis arguments in [4], but make an essential use of almost holomorphic extensions, introduced by Hörmander [3] and others. See [3] with the attached commentary and additional bibliography by one of us. Almost holomorphic extensions play an important role in the global theory of Fourier integral operators with complex phase [6]. Complex phase arguments were also used by A. Laptev, Yu. Safarov, and D. Vassiliev [5] in their approach to Fourier integral operators with real phase and their global representations as superpositions of Gaussian type operators and at one point the authors evoke almost analytic (i.e. almost holomorphic) extensions.

\section{Main result}

As in Section 5.2. in [4] we use Stokes' formula to get

$$
f(z, k)=\frac{1}{2 i \bar{k}} \int_{\partial \Omega} \frac{1}{z-w} e^{\overline{k w}-k w} \mathrm{~d} w+\frac{\pi}{\bar{k}} e^{\overline{k w}-k w} 1_{\Omega}(z) .
$$

We write $k w-\overline{k w}=(i / h) \Re(w \bar{\omega})$, with $h=1 /|k|, \omega=2 \bar{k} /|k|$.

Consider the function $i u_{0}(w)=k w-\overline{k w}$ on $\partial \Omega$ (suppressing sometimes the large parameter from the notation). In [4] we use the analyticity of $\partial \Omega$ to introduce a holomorphic extension $i u(w)$ of $i u_{0}(w)$ from $\partial \Omega$ to neigh $(\partial \Omega, \mathbb{C})$. 
When $\partial \Omega$ is only smooth we take instead an almost holomorphic extension $u \in$ $C^{\infty}(\operatorname{neigh}(\partial \Omega, \mathbb{C}))$ so that

$$
\left.u\right|_{\partial \Omega}=u_{0}, \quad \partial_{\bar{w}} u(w)=\mathscr{O}\left(\operatorname{dist}(w, \partial \Omega)^{\infty}\right),
$$

see [6],[7]. We recall that $u$ is unique up to infinite order: If $\tilde{u}$ is another analytic holomorphic extension of $u_{0}$ then

$$
u-\tilde{u}=\mathscr{O}\left(\operatorname{dist}(\cdot, \partial \Omega)^{\infty}\right) \text { in neigh }(\partial \Omega, \mathbb{C}) .
$$

As in [4] eq. (5.13) we parametrize points $w$ in a neighbourhood of $\partial \Omega$ by

$$
w=\gamma(t)+\mathrm{i} s \dot{\gamma}(t), \quad t \in \mathbb{R} / L \mathbb{Z}, \quad s \in \operatorname{neigh}(0, \mathbb{R}),
$$

where $L>0$ is the length of $\partial \Omega$. Here $t \rightarrow \gamma(t)$ is a parametrization of $\partial \Omega$ with positive orientation and with $|\dot{\gamma}(t)|=1$, such that $\gamma$ can be viewed as an $L$-periodic function. As in [4] Section 5, we can view $u$ as a function $u=u(t, s), t \in \mathbb{R} / L \mathbb{Z}, s \in \operatorname{neigh}(0, \mathbb{R})$, satisfying

$$
\partial_{t} u+\mathrm{i}\left(1+\mathrm{i} s \frac{\ddot{\gamma}}{\gamma}\right) \partial_{s} u=\mathscr{O}\left(s^{\infty}\right),
$$

and with $u(t, s)=u_{0}(t)+s u_{1}(t)+s^{2} u_{2}(t)+\mathscr{O}\left(s^{3}\right)$, we get

$$
\begin{aligned}
& \partial_{t} u_{0}(t)=\frac{|k|}{2}(\dot{\gamma}(t) \bar{\omega}+\bar{\gamma}(t) \omega)=|k|\langle\dot{\gamma}(t), \omega\rangle_{\mathbb{R}^{2}}, \\
& \partial_{t}^{2} u_{0}(t)=\frac{|k|}{2}(\ddot{\gamma}(t) \bar{\omega}+\bar{\gamma}(t) \omega)=|k|\langle\ddot{\gamma}(t), \omega\rangle_{\mathbb{R}^{2}} .
\end{aligned}
$$

From (2.6) we see that $\gamma(t)$ is a critical point of $u_{0}(t)$ iff $\omega$ (which is non-vanishing) is normal to $\partial \Omega$ at $\gamma(t)$. From $\langle\dot{\gamma}(t), \dot{\gamma}(t)\rangle=1$ we know that

$$
\langle\dot{\gamma}(t), \ddot{\gamma}(t)\rangle=0,
$$

and hence $\ddot{\gamma}(t)$ is normal to $\partial \Omega$ everywhere. Thus at a critical point of $u_{0}$ we have $\ddot{\gamma}(t) \in \mathbb{R} \omega$. It follows from (2.7) that a critical point is non-degenerate precisely when $\ddot{\gamma}(t) \neq 0$, i.e. when $\partial \Omega$ has non-vanishing curvature there. Such a point is

- a local maximum if $\ddot{\gamma}(t)=c \omega, c<0$, and

- a local minimum if $\ddot{\gamma}(t)=c \omega, c>0$.

Now recall the assumption that

$\Omega$ is strictly convex.

Then at every point in $\partial \Omega, \ddot{\gamma}(t)$ is non-vanishing and of the form $c(t) v(t)$, where $c(t)>0$ and $v(t)=\mathrm{i} \dot{\gamma}(t)$ is the interior unit normal (recall that $\gamma$ is positively oriented).

For a fixed $k \neq 0$, we can decompose

$$
\partial \Omega=\left\{w_{-}(k)\right\} \cup \Gamma_{+} \cup\left\{w_{+}(k)\right\} \cup \Gamma_{-},
$$

ordered in the positive direction when starting and ending at $w_{-}(k)$. Here 
- $w_{-}(k)$ is the south pole, where $v=c \omega$ for some $c<0$. Equivalently this is the global maximum point of $u_{0}$.

- $\Gamma_{+}$is the open boundary segment connecting $w_{-}(k)$ to $w_{+}(k)$ in the positive direction.

- $w_{+}(k)$ is the north pole, where $v=c \omega$ for some $c>0$. Equivalently this is the global minimum point of $u_{0}$.

- $\Gamma_{-}$is the open boundary segment connecting $w_{+}(k)$ to $w_{-}(k)$ in the positive direction.

Notice that

$$
\Gamma_{ \pm}=\left\{\gamma(t) \in \partial \Omega ; \mp \partial_{t} u_{0}(\gamma(t))>0\right\}
$$

On $\partial \Omega$ we have :

$$
e^{\overline{k w}-k w}=e^{-i u_{0}(w)}, u_{0}(w)=|k| \Re(w \bar{\omega}), w=\gamma(t) .
$$

The formula (2.1) reads

$$
f(z, k)=\frac{1}{2 i \bar{k}} \int_{\partial \Omega} \frac{1}{z-w} e^{-i u(w, k)} d w+(\pi / \bar{k}) e^{-i|k| \Re(z \bar{\omega})} 1_{\Omega}(z),
$$

where $u(\cdot, k)$ is an almost holomorphic extension of $u_{0}(w)=u_{0}(w, k)$ to a neighbourhood of $\partial \Omega$.

We introduce the same closed contour $\Gamma$ as in [4], Subsection 5.3. Recall that $\Gamma$ is a deformation of $\partial \Omega$ obtained by pushing $\Gamma_{+}$inward and $\Gamma_{-}$outward. This is obtained by means of a smooth vector field $v$, defined near $\partial \Omega$, transversal to $\partial \Omega$ and pointing outward, so that

$$
\Gamma=\left\{\left.\exp (t v(w))\right|_{t=\tau(w)} ; w \in \partial \Omega\right\},
$$

when $\tau(w)$ is a suitable function such that $\sup |\tau| \ll 1, \tau<0$ on $\Gamma_{+}, \tau>0$ on $\Gamma_{-}$. Near $w_{+}$we can choose a real Morse coordinate $\mu$ on $\Gamma$, centred at $w_{+}$such that as a function of $\mu$,

$$
u=u\left(w_{+}(k), k\right)+|k| \mu^{2} / 2 .
$$

Let $\mu$ also denote an almost holomorphic extension. Then in a complex neighbourhood of $w_{+}$, we have $u$ as a function of $\mu$

$$
u=u\left(w_{+}(k), k\right)+|k| \mu^{2} / 2+|k| \mathscr{O}\left((\mathfrak{I} \mu)^{\infty}\right) .
$$

Near $w_{+}$we choose $\Gamma$ to coincide with $\exp (-\mathrm{i} \pi / 4) \mathbb{R}$ in the complex $\mu$-coordinate. Thus along this part of $\Gamma$, we have

$$
-\mathrm{i}\left(u(\cdot, k)-u\left(w_{+}(k), k\right)\right)=-|k||\mu|^{2} / 2+|k| \mathscr{O}\left(|\mu|^{\infty}\right) .
$$


Near $w_{-}$we have a similar construction. Along $\Gamma$ we have

$$
\left|e^{-i u(w, k)}\right| \leq e^{-|k| \operatorname{dist}\left(w,\left\{w_{+}(k), w_{-}(k)\right\}\right)^{2} / C} .
$$

Let $\Omega_{+} \subset \bar{\Omega}$ and $\Omega_{-} \subset \mathbb{C} \backslash \Omega$, be the points swept over by the deformation of $\Gamma_{+}$ and $\Gamma_{-}$respectively,

$$
\begin{aligned}
& \Omega_{+}=\left\{\exp t v(w) ; w \in \Gamma_{+}, \tau(w) \leq t \leq 0\right\}, \\
& \Omega_{-}=\left\{\exp t v(w) ; w \in \Gamma_{-}, 0 \leq t \leq \tau(w)\right\},
\end{aligned}
$$

and notice that

$$
\Gamma=\partial\left(\left(\Omega \backslash \Omega_{+}\right) \cup \Omega_{-}\right) .
$$

Assume for simplicity that $z \notin \partial \Omega_{+} \cup \partial \Omega_{-}$and put

$$
F(z)=F_{\Gamma}(z)=\int_{\Gamma} \frac{1}{z-w} e^{-i u(w, k)} d w .
$$

When $\Gamma$ is real-analytic, we can choose $u$ holomorphic and using the residue theorem we got in [4]

$$
\begin{aligned}
f(z, k) & =\frac{1}{2 i \bar{k}} \int_{\partial \Omega} \frac{1}{z-w} e^{-i u(w, k)} d w+(\pi / \bar{k}) e^{-i|k| \Re(z \bar{\omega})} 1_{\Omega}(z) \\
& =\frac{1}{2 i \bar{k}} F(z)+(\pi / \bar{k})\left(e^{-i u(z, k)}\left(1_{\Omega_{-}}(z)-1_{\Omega_{+}}(z)\right)+e^{-i|k| \Re(z \bar{\omega})} 1_{\Omega}(z)\right) .
\end{aligned}
$$

In the smooth case we get a correction term that we compute with the help of Stokes' theorem. Let $V \Subset \mathbb{C}$ have a smooth positively oriented boundary. If $g$ is a distribution defined near $\bar{V}$ sufficiently smooth near $\partial V$, Stokes' formula gives

$$
\frac{1}{2 \mathrm{i}} \int_{\partial V} g(w) \mathrm{d} w=\int_{V} \partial_{\bar{w}} g \frac{\mathrm{d} \bar{w} \wedge \mathrm{d} w}{2 \mathrm{i}}=\int_{V} \partial_{\bar{w}} g L(\mathrm{~d} w) .
$$

With $g(w)=\frac{1}{z-w} e^{-\mathrm{i} u(w, k)}$ and $\delta_{z}$ denoting the delta mass at the point $z$, we get

$$
\partial_{\bar{w}} g=-\pi \delta_{z}(w) e^{-\mathrm{i} u(z, k)}-\frac{\mathrm{i} \partial_{\bar{w}} u}{z-w} e^{-i u(w, k)},
$$

hence

$$
\frac{1}{2 \mathrm{i}} \int_{\partial V} \frac{1}{z-w} e^{-\mathrm{i} u(w, k) \mathrm{d} w}=-\pi e^{-i u(z, k)} 1_{V}(z)-\mathrm{i} \int_{V} \frac{\partial_{\bar{w}} u(w, k) e^{-\mathrm{i} u(w, k)}}{z-w} L(\mathrm{~d} w)
$$

We apply this with $V$ equal to $\Omega_{+}$and $\Omega_{-}$and get

$$
\begin{aligned}
& \quad \frac{1}{2 \mathrm{i}} \int_{\partial \Omega} \frac{1}{z-w} e^{-\mathrm{i} u(w, k)} \mathrm{d} w-\frac{1}{2 \mathrm{i}} \int_{\Gamma} \frac{1}{z-w} e^{-\mathrm{i} u(w, k)} \mathrm{d} w= \\
& -\pi e^{-i u(z, k)} 1_{\Omega_{+}}(z)+\pi e^{-\mathrm{i} u(z, k)} 1_{\Omega_{-}}(z)-\mathrm{i} \int \frac{\partial_{\bar{w}} u(w, k)}{z-w} e^{-\mathrm{i} u(w, k)}\left(1_{\Omega_{+}}(w)-1_{\Omega_{-}}(w)\right) L(\mathrm{~d} w)
\end{aligned}
$$


The last term gives rise to a new term in (2.17) and we get

$$
\begin{array}{r}
f(z, k)=\frac{1}{2 i \bar{k}} F(z)+(\pi / \bar{k})\left(e^{-i u(z, k)}\left(1_{\Omega_{-}}(z)-1_{\Omega_{+}}(z)\right)+e^{-i|k| \Re(z \bar{\omega})} 1_{\Omega}(z)\right) \\
+\frac{i}{\bar{k}} \int \frac{\partial_{\bar{w}} u(w, k)}{z-w} e^{-\mathrm{i} u(w, k)}\left(1_{\Omega_{-}}(w)-1_{\Omega_{+}}(w)\right) L(\mathrm{~d} w) .
\end{array}
$$

In order to estimate the last term we notice that

$$
\left|e^{-\mathrm{i} u(w, k)}\right| \leq e^{-|k| \operatorname{dist}(w, \partial \Omega) / C}, \quad w \in\left(\Omega_{+} \cup \Omega_{-}\right) \backslash \operatorname{neigh}\left(\left\{w_{+}, w_{-}\right\}\right) .
$$

In a neighbourhood of $w_{+}$, we use the Morse coordinates in (2.14) and notice that with $t=\mathfrak{R}(\mu), s=\mathfrak{I}(\mu)$ we have in

$$
\operatorname{neigh}\left(w_{+}\right) \cap \Omega_{-}: 0<t<1 / \mathscr{O}(1), \quad-t<s<0,
$$

and in

$$
\operatorname{neigh}\left(w_{+}\right) \cap \Omega_{+}:-1 / \mathscr{O}(1)<t<0, \quad 0<s<-t .
$$

In both sets, $-\mathfrak{I}(u)=-t s+\mathscr{O}\left(s^{\infty}\right) \geq s^{2}-\mathscr{O}\left(s^{\infty}\right)$, so

$$
\left|e^{-\mathrm{i} u(w, k)}\right| \leq e^{-|k|\left(s^{2}+\mathscr{O}\left(s^{\infty}\right)\right)} \text { in neigh }\left(w_{+}\right) \cap\left(\Omega_{-} \cup \Omega_{+}\right) .
$$

The same estimate holds near $w_{-}$in the corresponding Morse coordinate. Combining (2.19), (2.20), (2.2) we get

$$
\left|\left(\bar{\partial}_{w} u\right) e^{\mathrm{i} u(w, k)}\right|=\mathscr{O}\left(|k|^{-\infty}\right) \text { uniformly in } \Omega_{+} \cup \Omega_{-} .
$$

We conclude that the last term in (2.18) is $\mathscr{O}\left(<z>^{-1}|k|^{-\infty}\right)$.

The analysis of $F_{\Gamma}$ in [4] goes through without any changes and we obtain:

Theorem 2.1. The detailed description of the asymptotics of $f$ when $k \rightarrow \infty$ in [4, Theorem 5.2] remains valid if we replace the assumption that $\partial \Omega$ is real-analytic, by the weaker assumption that $\partial \Omega$ is smooth.

The results in [4, Subsection 5.5] about the leading approximations of $\phi_{1}, \phi_{2}$ in certain polynomially weighted $L^{2}$-norms remain valid after the same weakening of the boundary regularity assumption for $\Omega$.

Acknowledgments. This work is partially supported by the ANR-FWF project ANuI - ANR17-CE40-0035, the isite BFC project NAANoD, the EIPHI Graduate School (contract ANR17-EURE-0002) and by the European Union Horizon 2020 research and innovation program under the Marie Sklodowska-Curie RISE 2017 grant agreement no. 778010 IPaDEGAN. 


\section{References}

[1] A.P. Calderón, On inverse boundary value problem, Seminar on Numerical Analysis and its Applications to Continuum Physics (Rio de Janeiro, 1980) pp 65-73 (Soc. Brasil. Mat.)

[2] A.S. Fokas, On the Inverse Scattering of First Order Systems in the Plane Related to Nonlinear Multidimensional Equations, Phys. Rev. Lett. 51, 3-6 (1983)

[3] L. Hörmander, Fourier Integral Operators, Lectures at the Nordic Summer School of Mathematics, Tjörn 1969, with some comments by J. Sjöstrand 2018, https://portal.research.lu.se/portal/en/publications/fourier-integral-operators (7730

[4] C. Klein, J. Sjöstrand, N. M. Stoilov, On the large $|k|$ behavior of complex geometric optics solutions to d-bar problems, arXiv:2009.06909

[5] A. Laptev, Yu. Safarov, D. Vassiliev, On global representation of Lagrangian distributions and solutions of hyperbolic equations, Comm. Pure Appl. Math. 47(1994), no. 11, 1411-1456.

[6] A. Melin, J. Sjöstrand, Fourier integral operators with complex-valued phase functions, Fourier integral operators and partial differential equations (Colloq. Internat., Univ. Nice, Nice, 1974), pp. 120-223. Lecture Notes in Math., Vol. 459, Springer, Berlin, 1975.

[7] J. Sjöstrand, M. Zworski, Complex scaling and the distribution of scattering poles, Journal of the AMS, 4(4)(1991), 729-769. 